\title{
«Laissez venir à moi les femmes et les petits enfants »: la philogynie bien tempérée d'un prédicateur silésien au début du XVIe siècle
}

\author{
Hervé Martin
}

\section{OpenEdition Journals}

\section{Édition électronique}

URL : http://journals.openedition.org/abpo/1615

DOI : $10.4000 / a b p o .1615$

ISBN : 978-2-7535-1486-7

ISSN : 2108-6443

Éditeur

Presses universitaires de Rennes

Édition imprimée

Date de publication : 20 juin 2002

Pagination : 117-128

ISBN : $978-2-86847-742-2$

ISSN : 0399-0826

\section{Référence électronique}

Hervé Martin, « "Laissez venir à moi les femmes et les petits enfants » : la philogynie bien tempérée d'un prédicateur silésien au début du XVIe siècle ", Annales de Bretagne et des Pays de l'Ouest [En ligne], 109-2 | 2002, mis en ligne le 20 juin 2004, consulté le 23 avril 2019. URL : http:// journals.openedition.org/abpo/1615; DOI : 10.4000/abpo.1615 


\title{
" Laissez venir à moi les femmes et les petits enfants ": la philogynie bien tempérée d'un prédicateur silésien au début du XVI ${ }^{\mathrm{e}}$ siècle
}

\author{
Hervé MARTIN \\ Professeur émérite d'histoire médiévale - CRHISCO, université Rennes 2
}

Quelques précisions s'imposent d'entrée de jeu pour bien cerner la nature de mon intervention, dont le titre laisse clairement entendre qu'elle ne s'inscrit pas, loin s'en faut, sous le signe d'une peur panique de l'autre sexe. S'il s'agissait d'apporter un nouvel exemple de la crainte que les femmes inspiraient couramment aux moines, aux religieux et aux clercs médiévaux, et d'enrichir le florilège de la misogynie ordinaire, notre communication serait inutile. En effet, le dossier constitué sur ce sujet par les historiens est bien assez fourni, à commencer par les pages classiques de Jean Delumeau dans La peur en Occident, où une grande place est faite au réquisitoire du franciscain Alvaro Pelayo, grand pénitencier de la cour d'Avignon, contre le deuxième sexe. Dans le De planctu ecclesiae, composé vers 1330 à la demande du pape Jean XXII, ce théologien n'a pas recensé moins de 102 vices et méfaits attribuables à la femme ${ }^{1}$. J'ai choisi de faire entendre aujourd'hui un son de cloche différent, qui nous est fourni par le dominicain silésien Pérégrin d'Opole, un contemporain de Pelayo, né vers 1260 et décédé vers 1330. Sensible à la présence des femmes à ses sermons, il était soucieux de leur plaire, comme le montre cette appréciation qu'il prête à ses auditrices : "Grand bien à ce prédicateur, pour avoir si bien parlé en notre faveur contre les hommes. " L'expression " contre les hommes ", si forte soit-elle, ne doit pas nous induire en erreur : tout en épousant la cause des femmes battues et opprimées, et tout en leur prêtant des qualités et des aptitudes dont les hommes sont dépourvus dans le domaine spirituel, l'orateur se garde bien d'encourager la guerre des sexes et se montre soucieux de ne pas semer la zizanie dans les ménages ${ }^{2}$.

1. Delumeau, Jean, La peur en Occident, XIVe-XVIII siècles, Paris, 1978, p. 317-320.

2. La source de cette étude est un recueil de 128 sermons du temps (répartis sur l'ensemble de l'année liturgique) et des saints composés vers 1300 par le dominicain polonais Pérégrin d'Opole (vers 1260-vers 1330), un contemporain de maître Eckhart et de 
Avant d'entrer dans le vif du sujet, je vais essayer de cerner brièvement la vie, l'œuvre et le terrain d'action de Pérégrin. Né vers 1260, il a passé sa jeunesse à Opole et à Ratibor en Silésie, cette principauté relevant à l'époque du royaume de Pologne. Ratibor, à laquelle on attribue plus de 3000 habitants, a abrité une communauté de dominicains à partir de 1239, alors qu'Opole a dû attendre les années 1280 pour voir s'installer ces religieux. Le jeune Pérégrin a fait profession au couvent de Ratibor et y a fait ses études, avec succès, puisqu'il a été élu prieur de cet établissement en 1303. La même année, il est devenu le pénitencier de la famille ducale de Silésie, ce qui lui a valu de prononcer des homélies en polonais devant la cour. C'est vraisemblablement en ces années, entre 1297 et 1304, qu'il a composé ses sermons de tempore et de sanctis. En 1305, il est devenu provincial de Pologne et l'est resté jusqu'en 1312, à charge de veiller sur 35 couvents, dont 3 de religieuses. Après être rentré dans le rang et avoir enseigné au studium de Ratibor, Pérégrin a été promu aux fonctions d'inquisiteur en 1318 par le pape d'Avignon Jean XXII. Contemporain de Pelayo, l'un des hérauts de la misogynie cléricale, notre homme est aussi un discret émule du redoutable Bernard Gui, l'auteur du Manuel de l'inquisiteur, obsédé par la menace hérétique sous toutes ses formes. Dès 1322, Pérégrin est redevenu provincial de Pologne; la fin de sa vie, intervenue dans les années 1330 au couvent de Breslau, est mal connue.

Qu'il s'agisse de Breslau/Wroclaw, de Ratibor ou d'Opole, nous avons chaque fois à faire à des villes de locatio, disons, pour parler vite, à d'anciens noyaux de peuplement slaves régularisés dans le courant du XIII ${ }^{\mathrm{e}}$ siècle par des colons allemands, au point de prendre des allures de villes neuves. En tous ces lieux, régulièrement visités par le duc et sa cour, le commun peuple slave et les aristocrates polonais, promoteurs des opérations de locatio, cohabitaient avec des marchands et des artisans allemands, devant lesquels il fallait prêcher en langue germanique. Opole, bien connue grâce aux travaux de Dieter Veldtrup, avait également près de 3000 habitants, comme Ratibor, et bénéficiait d'un dense encadrement religieux : deux paroisses, une collégiale, un couvent de franciscains et un autre de dominicains. Dite " de droit allemand ", cette petite cité abritait une population mêlée, dont trois composantes sont assez bien connues grâce aux biogrammes de 900 individus qui y ont vécu entre 1200 et 1600. Il s'agit du clergé, des magistrats et des officiers urbains, et enfin du personnel de la cour ducale $^{3}$. Ils ne constituaient pas la totalité du public des sermons, dans lequel il faut faire une place aux artisans, aux maîtres des métiers et, évidemment, à leurs épouses.

Bernard Gui, dont l'existence s'est partagée entre Ratibor, Opole et Breslau/Wroclaw, trois villes germano-polonaises dites de locatio. Les sermons de Pérégrin d'Opole ont connu une grande diffusion aux XIV e et XV ${ }^{\mathrm{e}}$ siècles dans toute la Germanie et l'Europe centrale. Ils ont été édités à Varsovie en 1997 (650 pages).

3. VeldTRuP, D., Prosopographische Studien zur Geschichte Oppelns als herzoglicher Residenzstadt im Mittelalter, Berlin, 1995. Voir un compte rendu de cet ouvrage par Joseph Morsel, dans le Bulletin de la Mission Historique Française en Allemagne, 1996, p. 179-180. 
Un écho assez fidèle de la prédication de Pérégrin d'Opole nous est fourni par un corpus de 128 homélies latines, partagé entre 65 sermons " du temps ", prononcés au fil de l'année liturgique, et 63 sermons " des saints ", proférés à l'occasion de leurs fêtes. Ayant lu et traduit la totalité de la première série et les allocutions les plus significatives de la seconde, je pense être désormais en mesure de cerner le discours sur l'autre sexe de ce contemporain de maître Eckhart, dont on sait qu'il vivait environné de béguines. Sans échapper totalement aux vieux clichés de la misogynie cléricale, Pérégrin sait faire preuve de compréhension et de sympathie envers l'autre moitié de l'humanité, à laquelle il prête souvent un rôle positif dans le domaine religieux. Non content de célébrer le mariage comme le premier des sacrements, il s'efforce de promouvoir des relations équilibrées entre les époux et n'hésite pas à s'en prendre au despotisme marital. Quand il lui arrive de dresser le portrait de saintes femmes, il le fait d'une façon qui n'est ni mièvre ni convenue.

\section{Clichés et exempla misogynes}

Quand il puise chez ses devanciers, Pérégrin reprend et véhicule inévitablement des stéréotypes et des anecdotes misogynes, qui font partie du fonds commun de la culture cléricale. Comme le nombre de ces emprunts est assez restreint, on ne saurait accorder trop d'importance à ce versant du discours. Des Vitae Patrum, les célèbres Vies des pères du désert, provient par exemple l'histoire de ces deux femmes, mariées l'une et l'autre, qui réussirent l'exploit de vivre quinze ans ensemble sans jamais se disputer. Pérégrin y ajoute son commentaire : avant de chercher à vivre en paix avec l'ensemble de l'humanité, il faut, dit-il, y parvenir d'abord avec sa femme. " En effet, enchaîne-t-il, si tu commences à te disputer avec elle, jamais tu ne feras la paix ${ }^{4}$."

Notre religieux partage avec nombre de ses confrères le préjugé selon lequel la femme est plus sujette que l'homme à la possession diabolique. Une démoniaque, raconte-t-il, voulait se confesser à un frère prêcheur sans parvenir à proférer un traître mot. Elle n'y parvint qu'après avoir suivi le conseil de parler avec le cœur et surtout après avoir expulsé par la bouche une énorme grenouille ${ }^{5}$. Dans un registre voisin, il est admis que le diable a eu neuf filles, mais non neuf fils, de Concupiscence, sa chère épouse. Sur ces neuf filles, il a réussi à en marier huit : la Simonie avec les prélats et les clercs, l'Hypocrisie avec les moines et les faux religieux, la Rapine avec les chevaliers, l'Usure avec les bourgeois, la Ruse avec les marchands, le Sacrilège avec les paysans, la Paresse avec les ouvriers, l'Orgueil et la Coquetterie avec les femmes elles-mêmes. Reste la neuvième fille, la Luxure,

4. Peregrini de Opole, Sermones de tempore et de sanctis, Varsovie, édition Richard Tatarzynski, 1997, p. 126-127, lignes 123-132. Désormais cet ouvrage sera mentionné sous l'appellation Sermones.

5. Sermones, $3^{\mathrm{e}}$ dimanche de carême, p. 90 et suiv. 
qu'il n'a mariée à personne pour mieux la prostituer à toutes sortes de gens ${ }^{6}$. La leçon de cet exemplum très diffusé est des plus claires : en tous domaines, et surtout en matière sexuelle, l'incitation au péché vient de la femme.

Une autre anecdote verse dans une franche misogynie. C'est l'histoire d'un philosophe qui regrette devant un de ses amis d'avoir planté dans son jardin un arbre auquel ses trois épouses se sont pendues successivement. Et son ami lui réplique : « De quoi te plains-tu? Te voilà bien débarrassé! Donne-moi une bouture de cet arbre, pour que je la plante dans mon jardin, pour qu'elle devienne un arbre et pour que ma méchante femme s'y pende $^{7}$. " On devine les sourires entendus des hommes de l'assistance et les ricanements des défenseurs du pouvoir mâle dans l'Église. Là s'arrêtent les compromissions de Pérégrin d'Opole avec l'idéologie misogyne. Il paie son tribut à la mentalité ambiante, rien de plus, et il le fait essentiellement quand il emprunte des anecdotes à ses devanciers, en somme quand il se laisse prendre au piège de l'intertextualité.

\section{Exaltation du sentiment maternel et insistance sur le rôle positif de la femme dans le domaine religieux}

En d'autres passages, bien plus nombreux, le frère dominicain amorce une défense et illustration de la femme dans son double rôle de mère et de pilier de la morale chrétienne. Il ne s'interdit certes pas, par le détour d'un exemplum, de faire grief à une femme d'avoir oublié de veiller au salut de son défunt mari. Mais le reproche est formulé sans virulence et concerne aussi les enfants. Par ailleurs quand il évoque la coutume juive imposant aux hommes et aux femmes de se rendre et de se tenir séparément au Temple, il se contente d'observer sobrement que " cette coutume avait été instaurée pour des raisons d'honnêteté ${ }^{8}$ ". Il n'en tire pas argument pour estimer nécessaire de tendre entre les deux sexes une draperie montée sur une corde, dispositif adopté par certains de ses confrères pendant leurs sermons.

Aux yeux de notre frère prêcheur, le deuxième sexe est le dépositaire de la fonction maternelle, qu'il apprécie évidemment en termes positifs. "Les bonnes mères, observe-t-il, ont cette habitude quand elles punissent leurs enfants et quand elles les corrigent au nom de la discipline : si elles voient qu'ils restent inconsolables, elles les apaisent par de douces paroles et elles les caressent ${ }^{9}$. " Cette remarque, qui ne va pas dans le sens des thèses de Philippe Ariès, est plus banale que le fait d'attribuer au Christ des comportements maternels. Le Sauveur, estime le prédicateur, ne cesse

6. Ibidem, p. 90 et suiv.

7. Voir WolnY, J., Exempla z kazan niedzielnych Pregryna z Opola, GEREMEK, Bronislaw, (éd.), Kultura elitarna a kultura masowa, Wroclaw, 1978, p. 250-251. Cet exemplum est signalé en note par Tatarzynski, Sermones, p. 189-190.

8. Sermones, p. 43.

9. Sermones, p. 111, l. 1 et suiv. 
de penser à nous au ciel, comme une mère s'inquiète en permanence de son fils parti au loin. De façon plus explicite encore, l'Homme-Dieu consolera les affligés comme " la mère cajole son fils qui pleure, d'abord en baissant la voix, ensuite en essuyant ses larmes, enfin en lui attribuant des douceurs [litt. des fruits] ou des récompenses. "Jésus agit bel et bien as a mother, pour reprendre le titre d'un ouvrage célèbre. Cette impression est confirmée plus loin, quand Jésus se porte au secours du pécheur comme une mère dont l'enfant " s'apprête à tomber dans la boue, dans l'eau ou dans le feu; elle accourt aussitôt et elle le prend pour l'empêcher de tomber $^{10}$ ". À faire du Christ une mère, on tranche l'épineux et insoluble problème de savoir si Dieu était Homme ou Femme, ou les deux à la fois.

L'autre volet du plaidoyer pro muliere qui se développe au fil des sermons consiste en cinq exempla où la femme joue un rôle positif dans l'optique cléricale. Dans le premier cas se trouve saluée la constance d'une femme de mineur qui fit " parvenir chaque jour du pain et du vin à son mari que la terre avait enseveli alors qu'il creusait pour en extraire de l'argent. Elle le sustenta pendant un an, au terme duquel on le délivra sain et sauf ${ }^{11}$ ". En quatre autres circonstances, la femme abreuve son pécheur de mari de bons conseils, pas toujours écoutés, et elle ne ménage pas ses efforts pour le remettre dans le droit chemin.

La première de ces épouses exemplaires est mariée à un bon bourgeois qui, le dimanche, au lieu d'aller à la messe, "montait à cheval pour aller visiter ses propriétés, ses moissons dans les champs ou ses moulins. Comme son épouse, qui était une femme bonne, l'avertissait de ne pas se comporter ainsi et d'aller au moins à l'église les jours fériés pour entendre prêcher des choses édifiantes, il lui répondit : " Je sais mieux ce que je dois faire que tes prédicateurs. "Faut-il préciser que ça se termine mal pour le mari ${ }^{12}$ ? Au lieu de raconter la fin, je souligne l'expression " tes prédicateurs ", lourde de sens.

La seconde est la femme d'un marchand en retraite, un pécheur endurci qui refuse obstinément de se confesser. "Enfin elle vit deux frères passer devant sa maison, qu'elle appela pour leur dire : "Voyez, chers frères, si vous pouvez de quelque façon décider mon époux à se confesser." "L'un des religieux y parvient, en usant d'un stratagème habile ${ }^{13}$. Rien n'eût été possible sans l'intervention de l'épouse.

La troisième à entrer en scène sur notre petit théâtre est une princesse dont le mari ne veut pas non plus se confesser, par crainte de la pénitence à accomplir. Il tourne en dérision la crédulité de son épouse : " "Et tu crois, toi, que l'enfer existe? Les Prédicateurs ont inventé tout cela pour qu'on les craigne." Ce que voyant, parce que cela ne servait à rien, sa femme le laissa en paix. Et une nuit, alors que le prince reposait sur sa couche, les

10. Sermones, p. 125-126, l. 97-101 ; p. 144-145, l. 56-66 ; p. 196, l. 80-84.

11. Sermones, p. 95, 1. 55-57.

12. Sermones, p. 44, 1. 23-36.

13. Sermones, p. $171,1.66$ et suiv. 
démons vinrent le prendre pour le porter en enfer. Voyant cela, son épouse, parce qu'elle était une sainte femme, pria le Seigneur de lui révéler la condition de son époux. Et voici que le matin, au lever, elle découvrit le corps de son mari tout noirci par des tisons ${ }^{14}$. " Plutôt que le châtiment du prince sceptique et trop sûr de lui, je retiendrai l'annonce faite à sa femme, érigée au rang de médiatrice entre terre et ciel.

Le dernier cas est celui de l'épouse d'un avocat véreux, hostile aux intérêts ecclésiastiques. "Frappé par la maladie, il commença à désespérer, mais sa femme, qui avait été dévote, alla discrètement trouver les religieux, à l'insu de son mari, et elle leur demanda de prier Dieu " pour qu'il exprime une vraie contrition. Les prières des frères s'avérèrent efficaces ${ }^{15}$.

Au moins deux de ces histoires révèlent une étroite connivence entre les femmes, d'un statut social enviable, et ceux qui sont appelés ou les frères, ou les prédicateurs ou les religieux, dans lesquels il n'est pas difficile de reconnaître les dominicains, autrement dit les confrères de Pérégrin d'Opole. Ne nous y trompons pas : ces épouses dont le rôle est bénéfique se plient à des modes de comportement directement inspirés de la vie monastique. Elles font partie des " corps dociles " chers à Michel Foucault. Leur portrait est dressé un peu plus loin, quand se trouve campée " la femme honnête, humble, dévote et pacifique. Elle ne se dispute jamais avec ses voisines, elle ne juge personne, elle ne rabaisse personne, elle ne commère pas sur les places, elle ne participe pas aux rondes. " À un niveau de mérite supérieur se situe la béguine ou la pénitente promise au salut éternel : « Et toi, pauvre femme, qui maintenant te tiens dans un vêtement gris, tu seras, grâce à ton humilité, reine dans le royaume des cieux ${ }^{16}$. " L'idéal féminin ultime de notre dominicain est bien celui de la mulier religiosa, de la femme seule confite en dévotions et en abstinences, même s'il doit s'accommoder d'une société où les mariés sont largement majoritaires.

\section{Célébration du mariage et défense de la femme mariée}

Peu de prédicateurs ont poussé aussi loin que Pérégrin d'Opole la célébration de la grandeur du mariage, la défense des droits de l'épouse et les attaques contre les maris tyranniques. C'est là qu'intervient la notion de philogynie bien tempérée que j'ai choisi de mettre en exergue.

Dans un sermon consacré aux noces de Cana, prononcé le deuxième dimanche après l'Épiphanie, l'orateur célèbre avec élan la dignité du mariage en proclamant que c'est " une règle que Dieu lui-même a instaurée, alors que les saints ont instauré les autres règles de vie ", qu'il énumère ensuite. "Saint Bernard a institué l'ordre griseus (écru), Benoît l'ordre noir, Dominique celui des Prêcheurs, etc., mais seul l'ordre du mariage a été institué par Dieu. Heureux ceux qui ont un tel abbé pour une telle

14. Sermones, p. 258, 1. 35-49.

15. Sermones, p. 273, 1. 107-116.

16. Sermones, p. 276, 1. 85-90; p. 222, 1. 15-17. 
dignité. Parmi les sept sacrements, il est unique et le premier, parce qu'il a été institué d'abord. " En somme, Adam et Ève, drapés dans leur candide nudité, renvoient toutes les congrégations religieuses au vestiaire! « La seconde dignité du mariage, poursuit le frère dominicain, tient au fait qu'il a été institué dans le temps le plus heureux qui fût jamais, avant le péché, avant que l'homme eût péché, pour signifier que l'homme qui le veut peut bien vivre dans le mariage, sans péché17. " Autant dire que les relations homme-femme peuvent retrouver leur innocence première et leur transparence d'avant la faute.

Emporté par son élan, Pérégrin ajoute que le mariage " a été institué dans le lieu le plus digne et les plus heureux qui soit au monde, c'est-à-dire au paradis... pour signifier que les individus mariés peuvent parvenir au paradis supérieur, entendons au royaume des cieux, à condition d'avoir bien vécu. " Si l'on comprend bien, le passage par le purgatoire n'est plus nécessaire aux conjoints pour se laver de l'impureté associée à l'activité sexuelle. Et notre religieux clôt son premier mouvement en soulignant que " le Seigneur a proclamé spécialement cet état heureux, bon et saint. Les hérétiques se trompent en effet quand ils disent qu'aucun homme ne peut se sauver dans le mariage. Le Seigneur a complètement ruiné leur opinion en venant lui-même aux noces en compagnie de sa Mère et de ses disciples, et en les honorant d'un signe solennel ", qualifié un peu plus loin de signe miraculeux ${ }^{18}$. Cette fois, on perçoit le sens de cette défense et illustration de la vie conjugale : il s'agit de combattre les courants hétérodoxes et hérétiques hostiles au mariage. Je pense qu'il s'agit de vaudois, de frères apôtres ou d'adeptes du Libre-Esprit, plutôt que de cathares, en voie de disparition au début du XIV ${ }^{\mathrm{e}}$ siècle.

Quelles que soient les arrière-pensées du prédicateur, on est frappé par cette exaltation sans réticence du mariage, difficile à concevoir si, comme le pensait Georges Duby, ce sacrement n'avait à l'époque qu'un siècle et demi d'existence. En fait, depuis l'époque paléochrétienne, l'union de l'homme et de la femme a été placée sous le regard de Dieu et avalisée par la communauté chrétienne. Au temps des moines et des chevaliers, le rituel s'est simplement précisé et étoffé. Si l'on adopte ce point de vue, le dithyrambe qui précède nous surprend moins, puisqu'il s'inscrit au terme d'un long processus et qu'il faut parer à la menace que font planer sur la société chrétienne les adeptes de la vie en solo, entendons de la vita apostolica sans règle bien définie.

L'autre volet du discours de Pérégrin vise à définir " une façon juste et légitime " de se comporter dans le mariage, à commencer par l'affection que doivent se prodiguer les époux. " Tu dois aimer ta femme, enjoint le prédicateur au mari, au point de ne jamais lui adresser de vilaine parole et de ne jamais la frapper. " Après cette recommandation élémentaire, il évoque très concrètement le cas de ceux qui, " après avoir passé du temps

17. Sermones, p. 48-52, 1. 13-19, 1. 20-23.

18. Ibidem, p. 49, l. 24-37. 
à la taverne, sont si furieux qu'ils seraient prêts à se battre. "Une seule chose les retient : la crainte de recevoir une raclée. Mais, de retour chez lui, l'homme humilié a tendance à se rattraper sur son entourage. "Lorsqu'il revient furieux à la maison, il retourne sur sa femme toute l'humiliation qu'il a subie à la taverne. Il la prend par les cheveux et la traîne d'un coin à l'autre de la maison. "L'orateur condamne vivement ces agissements au nom de l'amour dû à celle qui a quitté père et mère pour suivre son mari, et il revendique ensuite pour la femme des droits ou, à tout le moins, des avantages égaux à ceux de l'homme. "Il faut aimer ta femme, souligne-t-il, au point qu'elle doit avoir en tout les mêmes avantages que toi, pour le vêtement, la nourriture, la boisson et les autres commodités de l'existence. Mais je crains qu'il y en ait beaucoup qui ne tolèrent pour leurs femmes à peu près aucune propriété matérielle. Ils mettent tout sous clef devant elles, au point qu'elles n'ont le nécessaire ni pour leurs propres enfants ni pour elles-mêmes, par exemple pour payer le bain et autres choses. Quel conseil leur donner ${ }^{19}$ ?"

Le moraliste répond par le détour d'une fable consacrée à l'écureuil. Quand l'hiver approche, ce rongeur cherche avec sa femelle un arbre creux où entreposer des noix, en prévision des temps difficiles. "Quand la neige tombe, quand ils ne peuvent rien trouver à manger... le mâle court au trou où reposent les noix, et il se met à les ronger et à les manger ", sans laisser entrer sa femelle. Cette dernière cède à la force et " se dirige discrètement vers les racines de l'arbre... elle creuse par en-dessous, jusqu'à ce qu'elle parvienne aux noix. Alors, elle commence à le surveiller et, quand il ronge au-dessus, elle ronge en-dessous. " La sympathie du narrateur va très nettement à la femelle, qualifiée de pauvresse et contrainte de ruser pour ne pas mourir de faim ${ }^{20}$. Une autre version de la fable, donnée par Wolny, se clôt par la leçon suivante : "Ainsi certains se comportent-ils en voleurs de leurs épouses ${ }^{21}$. " Dans les deux cas, le prédicateur enchaîne : " Je ne vous apprends rien, mesdames, cependant je vous interdis de faire de grands trous" (dans les provisions, dans la caisse, comme on voudra). Autrement dit : à cette violence matérielle qui vous est faite, sachez répliquer habilement et avec modération. Bien conscient de la hardiesse de son propos, le frère prête à ses auditrices la réaction admirative dont j'ai fait état plus haut : "Grand bien à ce maître [à ce prédicateur selon Wolny], pour avoir si bien parlé pour nous et contre les hommes. "Cette réflexion, qui relève du vraisemblable énonciatif, en dit long sur les tensions hommes-femmes en milieu urbain.

Dans la même veine, le frère s'attaque à un autre problème, tout aussi délicat : jusqu'à quel point les femmes doivent-elles résister à leurs maris? Sans vouloir déclencher la guerre des sexes, il est loin de prôner la résignation et la soumission en toute circonstance. "Écoutez-moi, poursuit-il,

19. Sermones, p. 49-50, 1. 38-60.

20. Ibidem, p. 50, 1. 61-77.

21. Wolny, J., Exempla, op. cit., p. 250. 
je veux vous dire d'autres choses nouvelles. Il peut arriver parfois que la femme mérite de recevoir un soufflet de la part de son mari, sans parler de cas plus graves. Mais j'espère qu'il n'y a pas de femmes en ce lieu qui ne veulent jamais s'opposer en parole à leurs maris et qui font toujours ce qui plaît à ces derniers. Il y en a, en revanche, qui font toujours le contraire de leurs maris et qui revêtent ce manteau que l'on appelle nevesci swar, la querelle féminine, ou vrowencrik/frauwen Krig, la guerre des femmes. "Ce manteau, nous est-il précisé ensuite, est plus résistant que l'écarlate et peut rester dix ans sans se déchirer. Or certaines femmes " sont tellement bien revêtues de ce drap que, si leur mari leur dit : "Cette chose est blanche comme la neige", elles répliquent : "elle est noire comme du charbon", et le contraire le cas échéant. Je ne dis pas que ce soit votre cas ", s'empresse de préciser l'orateur, visiblement à la recherche d'une voie moyenne ${ }^{22}$. Tout en condamnant la guerre des sexes et la guérilla conjugale permanente, tout en dénonçant les ravages de l'esprit de contradiction, il estime que les femmes doivent savoir résister à leurs époux en certaines circonstances.

Si l'on se replace dans le cadre du "mâle Moyen Âge " et de la très phallocratique Église romaine, il ne me paraît pas excessif de voir en Pérégrin d'Opole un défenseur de la cause des femmes et des enfants agrippés à leurs cottes ou pendus à leurs mamelles. À preuve, la virulence avec laquelle il s'en prend aux tyrans domestiques : " L'homme coléreux, observe-t-il, grogne en sa maison comme l'ours, tantôt contre ses enfants, tantôt contre sa femme et sa domesticité, tantôt contre ses voisins. Et jamais son cœur ne s'apaise; il garde la colère dans son cœur un an, deux ans, dix ans, jusqu'à ce qu'il se venge de quelqu'un ${ }^{23}$. " Le prédicateur revient peu après à la charge contre ces tyranneaux par le détour d'un exemplum qui lui donne l'occasion de s'apitoyer sur une femme battue mais criminelle. Cette femme " avait un mari très méchant à son égard. Qu'il revînt de la messe, du marché ou de la taverne, chaque fois il la battait. " Se voyant dans une situation critique, la femme s'en alla consulter une sorcière, par l'entremise de laquelle elle conclut un pacte avec le diable afin de regagner les faveurs de son mari. Le prince des ténèbres y consentit à condition qu'elle sacrifiât son fils unique et qu'elle reniât le Christ et ses saints. Ce pacte ne l'empêcha pas, revenue à la maison, d'y subir les pires traitements. Une tentative de fuite, sur les détails de laquelle je passe, accrut la fureur de son tyran de mari, qui la laissa " étendue à demi-morte, couverte de blessures. " Elle réclama un confesseur à cor et à cri, et, malgré l'obstruction de son époux, elle parvint à faire l'aveu de ses énormes péchés, ce qui lui ouvrit les portes du paradis. J'ai dû résumer cette histoire à rebondissements. Si on la lit intégralement, on s'aperçoit que le mauvais rôle revient au mari, alors que la femme est une pauvresse prête à tout pour échapper à une situation intenable. Ses péchés sont pardonnables pour peu qu'elle se confesse en toute contrition ${ }^{24}$.

22. Ibidem, p. $250 ;$ Sermones, p. 50, 1. 77-85

23. Sermones, p. 293, 1. 64-68.

24. Sermones, p. 299-300, 1. 88-127. 
Tout en sachant que l'orateur n'est sans doute pas l'auteur de cette anecdote et qu'il s'est contenté de la rediffuser, on peut le créditer d'une certaine attention portée au sort des femmes battues ou victimes des plus sinistres machinations. Un autre exemplum a pour héroïne une femme délaissée par un mari infidèle, contrainte, pour survivre, de nourrir l'enfant d'un chevalier. Son mari s'en vient égorger le bambin, dont l'assassinat est imputé à la nourrice. Cette dernière échappe à la peine capitale grâce à l'intervention de la Sainte Vierge, alors que le vrai coupable périt misérablement, attaché à la queue d'un cheval ${ }^{25}$. En ce récit comme en d'autres, il s'agit d'abord de faire éclater la toute-puissance de la Sainte Vierge, mais le prédicateur n'en laisse pas moins percer une réelle sympathie pour les femmes délaissées et pour les enfants dont elles ont la charge.

\section{Des penchants féministes?}

Faut-il aller plus loin et prêter à Pérégrin d'Opole des penchants féministes avant la lettre? Pour avancer une réponse définitive, il faudrait prendre en compte l'ensemble des sermons qu'il consacre aux saintes reconnues par l'Église, où il faudrait d'ailleurs faire le tri entre ses très nombreux emprunts à la Légende dorée et les rares passages de son cru. Dans l'état actuel de mon enquête, je me limiterai à quelques brèves observations sur les homélies consacrées à deux saintes très vénérées en Europe centrale, sainte Élisabeth de Hongrie et sainte Hedvige de Silésie. Notre dominicain puise dans leurs Vies respectives pour bâtir ses allocutions, mais le choix et l'agencement des anecdotes, ainsi que quelques remarques, lui appartiennent.

Élisabeth, née en 1207, était la fille du roi André II de Hongrie. En 1221, elle épousa le landgrave de Thuringe et de Hesse, Louis IV. Après le décès de ce dernier, elle se retira dans un hôpital qu'elle avait fondé à Marburg, où elle décéda en 1231 à l'âge de 24 ans. Cette princesse qui a cultivé toutes les formes d'abaissement et de mortification, y compris le baiser au lépreux, a su se comporter quand il le fallait en forte femme, en résistant à son confesseur dominicain, le très redoutable inquisiteur Conrad de Marburg († 1233), auquel elle avait pourtant fait vœu d'obéissance. " Après la mort de son époux, elle s'en vint demander au frère Conrad de lui permettre de mendier de maison en maison. Et comme il le lui refusait, elle répondit : "Eh bien! Je le ferai, parce que vous ne pouvez pas m'en empêcher." " Le jour même, elle en fit serment à l'église ${ }^{26}$. Dans cet affrontement, le frère mendiant s'efforce de préserver la dignité princière de sa pénitente, qui se refuse à entrer dans ces vils calculs.

Pérégrin célèbre avec encore plus d'enthousiasme les mérites de sainte Hedvige (1174-1243), épouse du duc de Silésie Henri le Barbu, décédée et inhumée à Breslau en 1243. En dressant le panégyrique de la patronne et

25. Sermones, p. 191-192, 1. 186-212.

26. Sermones, p. 541, l. 54-63. 
protectrice de son pays natal, notre religieux la crédite de deux vertus qui, souligne-t-il, "se retrouvent dans toutes les femmes, dont elles révèlent la nature, à savoir la charité miséricordieuse et la dévotion. Ces deux vertus, précise-t-il, se rencontrent plus chez les femmes que chez les hommes". Plus loin, après avoir décrit des charités qui n'en restaient pas au stade symbolique, le prédicateur enfonce le clou. "La seconde vertu qu'ont les femmes est la dévotion. De fait, les femmes sont plus dévotes que les hommes pour se rendre à la messe, aux sermons et pour faire toutes sortes de bonnes œuvres ${ }^{27}$. " Alors, me direz-vous, c'est conventionnel, c'est paternaliste, ça relève aussi de l'autosatisfaction, puisque Pérégrin salue les mérites des plus soumises de ses ouailles et se réjouit de leur goût pour ces sermons qu'il profère avec talent. En somme, il renvoie l'ascenseur à ses groupies et il trône satisfait au milieu de femmes habituées à boire ses paroles. Je vous concède toutes ces remarques critiques, mais, si l'on tient compte du lien très fort entre culture et religion en milieu urbain, je me demande si ces éloges décernés aux femmes ne jouaient pas en faveur de la promotion de ces dernières. Je reconnais toutefois que ces passages ont moins de portée que ceux consacrés aux droits de la femme mariée et au concret de la vie conjugale.

Il est temps de conclure. Comme mon titre le laissait entendre, je n'ai pas apporté grand'chose au dossier sur « la peur de l'autre sexe ». Tout au contraire, j'ai examiné un cas de philogynie bien tempérée, exception faite de quelques exempla misogynes. S'agit-il d'une voix isolée ou, au contraire, d'une des variantes d'un discours pro muliere assez largement répandu? À défaut de pouvoir répondre à cette question essentielle, je précise que les sermons de Pérégrin d'Opole ont été largement diffusés, repris et rabâchés de la Bohême à la Suisse au bas Moyen Âge. De leur plein gré ou " à l'insu de leur plein gré ", par simple psittacisme, un certain nombre de confrères ont repris les assertions du frère dominicain. Ces textes pleins de compréhension pour les femmes, où il serait toutefois prématuré de rechercher les premiers linéaments d'un discours féministe, ont été composés dans un milieu urbain germano-polonais. On crédite habituellement les villes germaniques de cette époque d'un excédent de population féminine et d'un faible pourcentage de femmes mariées, générateurs l'un et l'autre d'un grand nombre de béguines ou assimilées. Ces femmes dévotes, ces mulieres religiosae, étaient avides de sermons et de biens spirituels de toutes sortes ${ }^{28}$. Au lieu de considérer ces femmes comme un danger pour le bon ordre matrimonial et comme de possibles concurrentes, au lieu de verser dans la crainte de l'autre sexe comme ce religieux italien qui traitait Claire de Rimini de possédée ${ }^{29}$, Pérégrin a su reconnaître leurs mérites et a su prendre leur défense contre le pouvoir despotique de leurs maris, cam-

27. Sermones, p. 619 , 1. 11-14; p. 625, 1. 83-85.

28. Voir Martin, Hervé, Mentalités médiévales, Paris, 1996, p. 416, 423-424.

29. Idem, Mentalités médiévales II, Paris, 2001, p. 74-75. 
pés sous un jour peu favorable. On croit déceler les signes d'une concurrence entre le guide spirituel attitré de ces dames et les époux possessifs et avares, encore qu'aucun passage ne le dise explicitement. Sans mettre en doute les bonnes intentions de notre moraliste, que j'aurais tendance à rapprocher de maître Eckhart et non plus de Bernard Gui ou d'Alvaro Pelayo, je rappelle toutefois que sa philogynie sert sa réputation de prédicateur, en lui attirant les faveurs d'une bonne moitié de la population. Somme toute, son discours relativement ouvert à la cause du deuxième sexe relevait de l'intérêt bien compris.

\section{RESUME}

Le dominicain polonais Pérégrin d'Opole (vers 1260-vers 1320), dont la carrière s'est déroulée dans la région de Breslau en Silésie, nous a laissé un corpus de 128 sermons de tempore et de sanctis. Loin de verser dans la misogynie courante chez les clercs de cette époque, il fait preuve d'une certaine philogynie. Il reprend bien quelques clichés et quelques exempla hostiles aux femmes, mais il ne manque pas une occasion de célèbrer les mérites des épouses et des mères de famille, en prenant leur défense contre des maris tyranniques. Quand il exalte les vertus de sainte Elisabeth de Hongrie et de sainte Hedvige de Silésie, on peut même taxer le prédicateur de discrets penchants féministes.

\section{ABSTRACT}

The polish dominican father Peregrinus de Opole (1260-1330), who has lived in the country of Breslau, has written a corpus of 128 sermons "de tempore et de sanctis". In same "exempla", the women are described as vicious and sinners, but in several sermons Peregrinus celebrates the good mothers who protect their children and the pious wifes who compel their husbands to go to church and to receive the sacrament of penance. The preacher thinks also that the wifes deserve the same rights and the same benefits as their husbands in the daily life. He blames strongly the men who hit their women when they are drunk. He praises the virtues of the holy women Elisabeth of Hungary and Hedvige of Silesy with enthusiasm. 